\title{
Bionanocomposites based on mesoporous silica and alginate for enhanced drug delivery
}

\author{
Hugo H.C. de Lima ${ }^{a}$, Vicente L. Kupfer ${ }^{\mathrm{a}}$, Murilo P. Moisés ${ }^{\mathrm{a}, \mathrm{d}}$, Marcos R. Guilherme ${ }^{\mathrm{b}}$, \\ Jaqueline de C. Rinaldi ${ }^{a, c}$, Sérgio L. Felisbino ${ }^{c}$, Adley F. Rubira ${ }^{b}$, Andrelson W. Rinaldi ${ }^{a, *}$ \\ ${ }^{a}$ Materials Chemistry and Sensors Laboratory - LMSen, State University of Maringá - UEM, 5790 Colombo Avenue, 87020-900, Maringá-PR, Brazil \\ ' State University of Maringá, UEM, 5790 Colombo Avenue, 87020-900, Maringá-PR, Brazil \\ ${ }^{\mathrm{c}}$ Laboratory of Extracelular Matrix-LabMEc, São Paulo University- UNESP, Botucatu-SP, Brazil \\ d Federal University of Technology of Paraná-UTFPR, 635 Marcílio Dias Street, 86812-460, Apucarana, PR, Brazil
}

\section{A R T I C L E I N F O}

\section{Keywords:}

Biomaterials

Cytotoxicity

Drug delivery

Hydrogel

Mesoporous silica

Nanotechnology

\begin{abstract}
A B S T R A C T
This work reports the preparation, the characterization and the prednisolone release profile of biocompatible hydrogel nanocomposites containing mesoporous silica (SBA) and alginate as a biomaterial for enhanced drug delivery with reduced burst effect and improved mechanical properties. Such systems, which were prepared using specific SBA/alginate-crosslinking chemistry, exhibited interconnecting pore hybrid network owing to both mesoporous silica and hydrogel characteristics. Activated SBA was shown to be a determinant factor in inhibiting initial burst by nearly $90 \%$ and the drug was released with minimal burst kinetics. The nanoparticles reduced the movements of polymer chains, affecting macromolecular relaxation, and the distribution of mesoporous silica within the hydrogel made drug release into surrounding liquid less favorable. The proposed systems are biocompatible with human immortalized RWPE-1 prostatic epithelial cells. This report offers an approach of up-to-date interest for the development of advanced biomaterials for further physiological and pathological applications.
\end{abstract}

\section{Introduction}

Hydrogels (HGs) are formed of 3D hydrophilic cross-linked polymer networks with ability to absorb and retain a large amount of water without their 3D structure being unmade (Ahmed, 2015; Din et al., 2015; Ghorpade, Yadav \& Dias, 2016, Hoare \& Kohane, 2007). Natural polymer-inspired HGs exhibit low toxicity, excellent biocompatibility, but they have the disadvantage of being more susceptible to mechanical stress, which can lead to ruptures inside the network, than the conventional materials (Huang, Liu, Liu, Liu \& Chen, 2014; Hou, Nie, Du, Xiong \& Fu, 2015). To prepare a HG with enhanced mechanical properties, many innovative approaches to achieving such an effect have been exhaustively used and many of them have already been implemented in the last decades. Approaches to this purpose have included the use of ceramics (Iviglia et al., 2016) and phosphate (Bastakoti et al., 2013) to form nanocomposites and synthetic polymers (Hou et al., 2015) to obtain copolymers or (IPN) interpenetrating polymer networks.

Currently, there is a deal great of interest in preparing HG-based nanocomposites owing to their biomedical relevance (Kerh, 2016;
Yadollahi et al., 2016). Such a system shows better biological and thermal, mechanical proprieties than pure HG did, making nanocomposite an ideal candidate for use in the biomedicine and pharmacy, such as controlled release and targeted delivery of drugs. Inorganic nanoparticles can minimize the burst release of HGs (high drug diffusion at early times) (Huang et al., 2015; Kerh, 2016; Silva et al., 2016). Materials which have been proven to be effective in the biomedicine include oxides (Liao et al., 2015; Hezaveh \& Muhamad, 2013; ZareAkbari et al., 2016), clay minerals (Gaharwar, Dammu, Canter, Wu \& Schmidt, 2011; Liang et al., 2012; Topuz, Bartneck \& Tacke, 2017; Wang et al., 2010), nanoparticles of silver (García-Astrain et al., 2015) and silica (Gaharwar, Rivera, Wu, Chan, \& Schmidt, 2013; Panic et al., 2015).

Santa Barbara Amorphous (SBA-15 and SBA-16) mesoporous silica offer a wide range of functional properties that can be brought into HGs. They Exhibit 2D hexagonal and 3D cubic structures with silanol groups that can be surface-modified with organic-ligands to form organic-inorganic hybrid materials, promoting multifunctional characteristics (Boza et al., 2016; Hashemikia, Hemmatinejad, Ahmadi \& Montazer, 2015). They have been shown to have relevant and specific

\footnotetext{
* Corresponding author.

E-mail addresses: hugolima22@hotmail.com (H.H.C. de Lima), awrinaldi@uem.br (A.W. Rinaldi).
} 
applications in adsorption (Shah, Din, Kanwal, \& Mirza, 2015), catalysis (Boza et al., 2016) solar cells (Chen et al., 2014) and drug delivery (Kamachi et al., 2016; Lian, Liang, Yamauchi \& Wu, 2011; Manzano et al., 2008; Sevimli \& Yilmaz, 2012) owing to their large specific area, narrow pore distribution, biocompatibility, and in vivo biodegradability. The entrapment of SBA inside HGs can lead to formation of optimized biomaterials that show better mechanical and release performances, opening a wider range of applications.

The preparation of HG nanocomposites based on SBA-15 and SBA16 for biomaterial-related applications requires the creation of a resistant, soft complex system free of harmful components so as not to affect their applications. The combination of SBA with a natural polymer can result in an advanced biomaterial whose properties can be modulated into a specific microenvironment. Alginate has received a great deal of attention as a biomaterial, owing to its advantages, such as biocompatibility and biodegradability (Poels et al., 2016). This polysaccharide is obtained from alginic acid which possesses several possible arrangements of chains because it has two different kinds of monomeric unities, both containing a carboxylic group in each. These segments are designated as L-mannuronic and L-guluronic acid. The alginate has been successfully used for many years in both the food and the beverage industries as thickening and gelling agents and also as a colloidal stabilizer. Commercial alginates are mainly obtained from three species of brown marine algae, such as Laminaria hyperborea, Ascophyllum nodosum and Macrocystis pyrifer. Other sources include Laminaria japonica, Eclonia maxima, Lesonia negrescens and Sargassum species. In all these species, the alginate is the main polysaccharide and corresponds up to $40 \%$ of the dry weight.

This work aimed at developing a hybrid HG nanocomposite as a biomaterial consisting of alginate and SBA-15 or SBA-16 in order to solve burst release-related drawbacks, besides improving mechanical properties, using prednisolone as a model drug. The prednisolone is a corticosteroid drug used for treating asthma, rheumatoid arthritis, ulcers, and multiple sclerosis, and for relieving the chemotherapy associated-symptoms in patients with cancer. In order to prepare a biomaterial that meet mechanical and biocompatible standards to intended applications, highly specific SBA/polymer-crosslinking chemistry was used. Approach shown here has included the biomaterial preparation in which SBA is tethered to alginate by covalent cross-linking, and a methodical characterization of HGs and SBA nanoparticles. Studies of the biological and physical-chemical properties of the final biomaterials as well as demonstration of their potential for drug delivery have been shown, with a view to relate structure/property to functionality for holding safety of intended applications.

\section{Materials and methods}

\subsection{Materials}

Pluronic P123 (99\%), Pluronic F127 (99\%), sodium alginate (50 $60 \mathrm{kDa}$; Mannuronate/Guluronate ratio $=1.42$ ), glycidyl methacrylate (GMA 97\%), tetraethyl orthosilicate (TEOS, 99\%), vinyltrimethoxysilane (VTMS, 99\%), 2-hydroxyethyl methacrylate (HEMA, 97\%,), N',N'-dimethylacrylamide (DMAAm, 97\%), sodium persulfate ( $\geq 98 \%$ ) and prednisolone $(\geq 99 \%)$ were purchased from Sigma-Aldrich. Toluene (99\%), hydrochloric acid $(37 \%, \mathrm{HCl})$ and ethanol (99\%) were supplied by Synth. Trypan Blue Solution (0.4\%), $10 \mathrm{mM}$ phosphate buffer (PBS) and antibiotics were purchased from Sigma Aldrich (St Louis, MO). Keratinocyte Serum Free Medium (K-SFM) and trypsinEDTA were obtained from (Gibco, Waltham, MA) and fetal bovine serum (FBS) was gotten from Invitrogen (Grand Island, NY, USA). All the reagents of analytical degree were used as received.

\subsection{Preparation of SBA-15 and SBA-16}

SBA-15 was prepared using the approach reported by Zhao, Feng et al. (1998). Briefly, $8.0 \mathrm{~g}$ of Pluronic P123 $\left(\mathrm{EO}_{20} \mathrm{PO}_{70} \mathrm{EO}_{20}\right)$ were added to an aqueous solution containing $240 \mathrm{~mL}$ of a hydrochloric acid solution and $30 \mathrm{~mL}$ of water at $40^{\circ} \mathrm{C}$ while stirring. After $4 \mathrm{~h}, 17.0 \mathrm{~g}$ of TEOS were introduced to stirred solution, which was left to react for $20 \mathrm{~h}$ at $40^{\circ} \mathrm{C}$. Then, the thus formed solution was brought into a Teflonlined stainless-steel autoclave which was kept in a stove at $80^{\circ} \mathrm{C}$ for $24 \mathrm{~h}$. The resulting material was filtered, washed with water, dried at $60{ }^{\circ} \mathrm{C}$ and burned at $550^{\circ} \mathrm{C}$ for $6 \mathrm{~h}$ with a heating rate of $1^{\circ} \mathrm{C} \mathrm{min}{ }^{-1}$. SBA-16 was obtained using a method proposed by Zhao, Huo, Feng, Chmelka, \& Stucky (1998). Two grams of Pluronic F127 $\left(\mathrm{EO}_{106} \mathrm{PO}_{70} \mathrm{EO}_{106}\right)$ were added to $80 \mathrm{~mL}$ of a $0.5 \mathrm{~mol} \mathrm{~L}^{-1}$ hydrochloric acid solution at $40^{\circ} \mathrm{C}$ to be dissolved. After $4 \mathrm{~h}$ of vigorous stirring, $8.4 \mathrm{~g}$ of TEOS were added to solution at $40^{\circ} \mathrm{C}$ while stirring. After $20 \mathrm{~h}$, the resulting solution was brought into the autoclave, kept in the stove at $80^{\circ} \mathrm{C}$ for $24 \mathrm{~h}$. The thus formed material was filtered, washed with water, dried $\left(60^{\circ} \mathrm{C}\right)$ and burned $\left(550^{\circ} \mathrm{C}\right)$ for $6 \mathrm{~h}$ with a heating rate of $1{ }^{\circ} \mathrm{C} \min ^{-1}$.

2.3. Preparation of mesoporous vinylsilica by surface functionalization of SBA-15 and SBA-16 with vinyltrimethoxysilane (VTMS)

One gram of VTMS and $1.0 \mathrm{~g}$ of SBA were added to $25 \mathrm{~mL}$ of toluene under stirring and the thus formed suspension was kept under reflux at $60{ }^{\circ} \mathrm{C}$ for $24 \mathrm{~h}$. After that, the final product was added to ethanol, centrifuged at $3000 \mathrm{rpm}$ and vacuum dried at $80^{\circ} \mathrm{C}$ for $8 \mathrm{~h}$. The surfacemodified materials were both labeled as SBA-15M and SBA-16 M to refer to mesoporous vinylsilica.

\subsection{Characterization of $S B A-15$ and $S B A-16$}

The structures of the mesoporous vinylsilica and silica were studied by small-angle X-ray diffraction (SAXS) patterns in a Bruker D8-advance equipped with a Cu- $\mathrm{K}_{\alpha}$ radiation $(\lambda=1.5406 \AA)$ by applying accelerating voltage of $40 \mathrm{kV}$, scanning speed of $0.005^{\circ} \mathrm{s}^{-1}$, and a current intensity of $30 \mathrm{~mA}$ in the intervals of $0.5^{\circ}<2 \theta>5.0^{\circ}$. The proprieties of texture were evaluated by elaborating physisorption isotherms at $77 \mathrm{~K}$ using a Quantachrome NOVA-1200E system and a Pore Analyzer. Data were processed with the use of Nova Win software. The surface area was measured by the Brunauer-Emmett-Teller (BET) method and the pore size distribution was determined by BJH BarrettJoyner Halenda approach. The removal efficiency of the structure-orienting agent was studied by means of FTIR spectroscopy with the use of a Perkin Elmer Spectrum Two system in the spectral range of $4000-$ $400 \mathrm{~cm}^{-1}$. A total of 128 scans were run for each spectrum to reach the resolution of $2 \mathrm{~cm}^{-1}$, using $\mathrm{KBr}$ pellets-diluted dry samples.

\subsection{Preparation of vinylated alginate}

The alginate was vinyl-functionalized using GMA as a chemical modifier convert it into a cross-linkable polysaccharide according to approach proposed by Reis et al., 2009. One gram of sodium alginate was added to $30 \mathrm{~mL}$ of water at $60^{\circ} \mathrm{C}$ while stirring. After homogenization, $2.0 \mathrm{~mol} \mathrm{~L}^{-1}$ hydrochloric acid solution was added dropwise until a pH 3.5 was obtained. After that, $0.65 \mathrm{~mL}$ of GMA was introduced and left to react at $60^{\circ} \mathrm{C}$ for $24 \mathrm{~h}$. Then, $150 \mathrm{~mL}$ of acetone were added to resulting solution for alginate precipitation. The formed solid, namely ALGMA, was filtered under vacuum, cooled with liquid nitrogen, and then freeze-dried for $24 \mathrm{~h}$. ALGMA was characterized by FTIR and nuclear magnetic resonance (NMR) spectroscopies with use of Varian spectrometer (model Mercury Plus BB) system at a frequency of 300.059 MHz for ${ }^{1} \mathrm{H}$ nucleus.

\subsection{Preparation of HG nanocomposites}

Known amounts of ALGMA, HEMA, DMAAm, SBA-15, SBA-15 M, SBA-16 and SBA-16M were added to $10 \mathrm{~mL}$ of water at room 
Table 1

Amounts of ALGMA, HEMA, DMAAm and mesoporous silica used in the HGforming reaction mixture.

\begin{tabular}{lllll}
\hline Samples & ALGMA (g) & HEMA (g) & DMAAm (g) & SBA (\% wt/wt) \\
\hline 025ALGMA & 0.25 & 0.5 & 0.25 & 0 \\
025ALGMA15 & 0.25 & 0.5 & 0.25 & 1.0 \\
025ALGMA15M & 0.25 & 0.5 & 0.25 & 1.0 \\
025ALGMA16 & 0.25 & 0.5 & 0.25 & 1.0 \\
025ALGMA16M & 0.25 & 0.5 & 0.25 & 1.0 \\
050ALGMA & 0.5 & 0.5 & 0.25 & 0 \\
050ALGMA15 & 0.5 & 0.5 & 0.25 & 1.0 \\
050ALGMA15M & 0.5 & 0.5 & 0.25 & 1.0 \\
050ALG16 & 0.5 & 0.5 & 0.25 & 1.0 \\
050ALG16M & 0.5 & 0.5 & 0.25 & 1.0 \\
\hline
\end{tabular}

temperature (Table 1) while stirring. An aqueous suspension of mesoporous vinylsilica and dissolved polymers was formed. Then, $10 \mathrm{mg}$ of sodium persulfate were added to stirred suspension at $60^{\circ} \mathrm{C}$ and the mixture turned to a transparent, soft material. The thus obtained hydrogels were washed with Milli- $\mathrm{Q}^{\circ}$ water to be characterized. The pure HG and the HG nanocomposites were labeled according to the synthesis compositions in Table 1.

\subsection{Swelling degree kinetics}

The swelling degree (SW) of the HGs were investigated by immersing the $1 \mathrm{~cm}^{3}$ dry samples of known weights into $250 \mathrm{~mL}$ of buffer solutions with $\mathrm{pH} 3.5,5.0$ and 7.4 at $37^{\circ} \mathrm{C}$. The samples were withdrawn from the solution buffer, the excess water droplets on the surface were wiped off carefully, and the samples were weighed at specific times until to achieve the swelling equilibrium. SW of HGs with different compositions was obtained from Eq. (1), correlating the water weight within the HG at any time $\left(\mathrm{W}_{\mathrm{s}}\right)$ to the initial weight of $\mathrm{HG}\left(\mathrm{W}_{0}\right)$.

$S D=\frac{W_{s}-W_{0}}{W_{0}} \times 100$

\subsection{Morphological properties}

Prior to SEM imaging, HGs were swollen to equilibrium in buffer solution of $\mathrm{pH}$ 7.4. They were withdrawn from water and immediately frozen by immersion in liquid nitrogen before being lyophilized for $24 \mathrm{~h}$. Under these conditions, it is supposed that the morphology of the swollen HGs is maintained. The samples were fractured and sputtercoated with a thin layer of gold and the SEM images were obtained in a scanning electron microscope (FEI-Quanta 250) by applying an acceleration voltage of $15 \mathrm{kV}$ and a current intensity of $30 \mu \mathrm{A}$.

\subsection{Mechanical properties}

Mechanical tests were considered as being the measured force for compressing the HGs to $1 \mathrm{~mm}$ deformation using a texture analyzerTAX.T2i equipped with a $5 \mathrm{~kg}$ load cell. The apparatus was equipped with a circular probe of $0.5 \mathrm{~mm}$ diameter $(\mathrm{P} / 0.5)$, which was programed to descend onto gel surface moving at a constant speed of $2 \mathrm{~mm} \mathrm{~s}^{-1}$. HGs were cut into pieces of $10 \mathrm{~mm}$ height and $100 \mathrm{~mm}^{2}$ of surface area. Their compressive strength was performed at $25^{\circ} \mathrm{C}$. The stress-strain measurements were done in triplicate, shortly after polymerization. Each measurement was performed in less than $1 \mathrm{~min}$ to prevent water loss from HG over the experiment. The force necessary for compressing the HGs at $1 \mathrm{~mm}$ was recorded and the stress values (s) were determined using Eq. (2) (Muniz \& Geuskens, 2001).

$\sigma=\frac{F}{A}=E\left(\lambda-\lambda^{-2}\right)$

where $\mathrm{F}$ is the force and A is the cross-sectional area of HG, E is the elastic modulus, and $\lambda$ is relative deformation of the sample $\left(\Delta \mathrm{l} / \mathrm{l}_{0}\right)$

\subsection{Drug release}

The prednisolone was loaded onto hydrogels by physical adsorption, which can be taken advantage for stimuli-triggered release of guest molecules from these systems. Prior to gelation, prednisolone (10\% wt/ $\mathrm{wt}$, with respect to the total mass of polymers) was introduced to the HG-forming mixture to be loaded during the synthesis. The samples were left to dry in a ventilated stove at $40^{\circ} \mathrm{C}$ until a constant mass was reached. After that, Prednisolone-loaded dry HGs of known weight were immersed into $250 \mathrm{~mL}$ of PBS buffer solution of $\mathrm{pH} 7.4$ at $37^{\circ} \mathrm{C}$ and stirred at $40 \mathrm{rpm}$ using a glass-made stirrer paddle. Aliquots of $3.0 \mathrm{~mL}$ were collected at specified times, and then absorption readings were made at $247 \mathrm{~nm}$, which is the wavelength for the maximum absorption of prednisolone, by means of an UV-vis spectrophotometer (Shimadzu, UV mini 1240). The aliquots were brought back into the reactor to prevent volume loss. The concentrations of prednisolone released from HGs were determined from analytical curves correlating the absorption to the concentration of prednisolone. In order to ensure the integrity of the release data, the dissolution kinetics of pure prednisolone in the PBS buffer with pH 7.4 was determined (supplementary data). The concentration of the drug in buffered solution was found to be constant with time, suggesting a long-term stability.

\subsection{Cell culture and cytoxicity assay}

Non-tumorigenic RWPE-1, a human papillomavirus 18 (HPV 18)

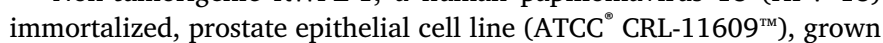
in Keratinocyte Serum Free Medium (K-SFM) supplemented with 1\% Lglutamine, antibiotics $\left(100 \mathrm{UI} / \mathrm{mL}\right.$ penicillin- $100<\mu \mathrm{g}>\mathrm{mL}^{-1}$ streptomycin), bovine pituitary extract, EGF plus $10 \% \mathrm{FBS}$, were grown and then distributed in a 24-well culture plate at $0.8 \times 105$ cells/well concentration and incubated in a humid atmosphere with $5 \% \mathrm{CO}_{2}$ at $37^{\circ} \mathrm{C}$ until $70 \%$ confluent monolayer be formed. Solutions containing synthetized materials were prepared in K-SFM with two different concentrations ( 1000 and $100<\mu \mathrm{g}>\mathrm{mL}^{-1}$ ). The cell medium was then removed from the 24 -well culture plate and $500 \mu \mathrm{L}$ of K-SFM containing the material was added in duplicate. The control cells were cultured in K-SFM without the addition of the material, only medium and named "vehicle". The plates were incubated for $48 \mathrm{~h}$. Then, cells were trypsinized by using trypsin-EDTA, washed out with phosphate buffer and centrifuged at $125 \mathrm{xg}$ for $5 \mathrm{~min}$. Subsequently, the cell pellets were resuspended in PBS and the cells were stained with Trypan Blue (TB) solution for $1 \mathrm{~min}$. The technique is based on the principle that live (viable) cells do not take up the dye, whereas dead (non-viable) cells do. The cell viability was calculated as the number of viable cells divided by the total number of cells within the grids on the Neubauer chamber under the phase contrast microscopy (Fig. S1). GraphPad Prism (version 5.00 for Windows; GraphPad Software, USA, www. graphpad.com) was used for statistical analysis. A significance level of $\mathrm{P} \leq 0.05$ was used.

\section{Results and discussion}

\subsection{Characterization of mesoporous silica}

Fig. 1 shows the XDR patterns of SBA-15, SBA-15 M, SBA-16, and SBA-16 M. In Fig. 1(A), there are three characteristic signs of diffraction in $2 \theta$ of $1.0323^{\circ}, 1.7421^{\circ}$ and $2.0228^{\circ}$ for SBA- 15 and in $2 \theta$ of $1.0266^{\circ}, 1.7307^{\circ}$ and $2.016^{\circ}$ for SBA-15 M. They are attributed to reflection planes (100), (110), and (200), respectively, which are associated to hexagonal, two-dimensional symmetry with a $\mathrm{p} 6 \mathrm{~mm}$ spatial group that indicates a mesostructure, as reported by Zhao, Feng et al. (1998) and Elumalai and Dharmalingam (2016). In Fig. 1(B), there are three characteristic diffraction peaks in $2 \theta$ of $0.8871^{\circ}, 1.06^{\circ}$ and 

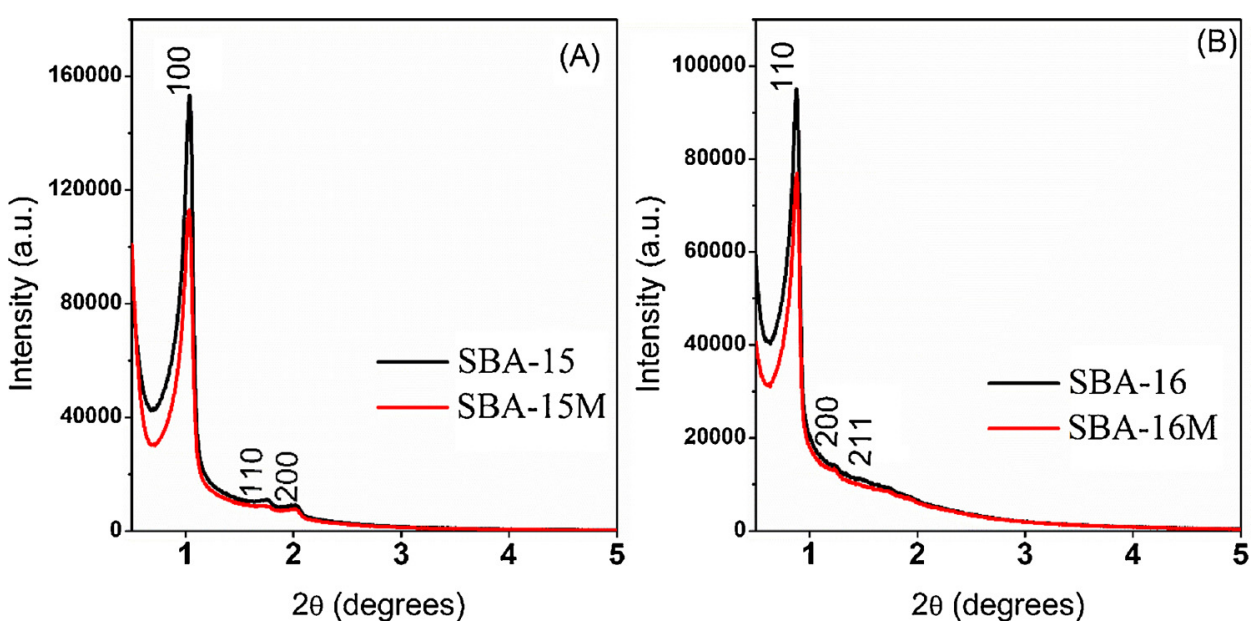

Fig. 1. XDR patterns of (A) SBA-15/SBA-15 M and (B) SBA-16/SBA-16 M.

Table 2

Textural properties of mesoporous silica.

\begin{tabular}{lllll}
\hline Samples & SBA-15 & SBA-15M & SBA-16 & SBA-16M \\
\hline $\mathbf{d}(\mathbf{n m})$ & $8.55^{\mathrm{a}}$ & $8.59^{\mathrm{a}}$ & $9.95^{\mathrm{c}}$ & $10.07^{\mathrm{c}}$ \\
$\boldsymbol{\alpha}_{\mathbf{0}}(\mathbf{n m})$ & $9.87^{\mathrm{b}}$ & $9.92^{\mathrm{b}}$ & $14.07^{\mathrm{d}}$ & $14.24^{\mathrm{d}}$ \\
$\mathbf{S}_{\text {BET }}\left(\mathbf{m}^{2} \mathbf{g}^{-\mathbf{1}}\right)$ & 700.56 & 534.42 & 667.5 & 475.48 \\
$\mathbf{V}_{\text {total }}\left(\mathbf{c m}^{\mathbf{3}} \mathbf{g}^{-\mathbf{1}}\right)$ & 0.78 & 0.65 & 0.48 & 0.33 \\
$\mathbf{V}_{\text {micro }}\left(\mathbf{c m}^{\mathbf{3}} \mathbf{g}^{-\mathbf{1}}\right)$ & 0.13 & 0.07 & 0.15 & 0.07 \\
$\mathbf{V}_{\text {meso }}\left(\mathbf{c m}^{\mathbf{3}} \mathbf{g}^{-\mathbf{1}}\right)$ & 0.65 & 0.58 & 0.34 & 0.27 \\
$\mathbf{D p}(\mathbf{B H J})(\mathbf{n m})$ & 5.05 & 4.43 & 3.59 & 3.59 \\
$\mathbf{W}(\mathbf{n m})^{*}$ & 4.82 & 5.49 & 10.48 & 10.65 \\
\hline
\end{tabular}

${ }^{\mathrm{e}} \mathrm{W}=\alpha_{0}-\mathrm{Dp}$.

a $\mathrm{d}(100)$

b $\alpha_{0}=\frac{2 d_{(100)}}{\sqrt{3}}$.

c $\mathrm{d}(110)$.

${ }^{\mathrm{d}} \alpha_{0}=\sqrt{2} d(110)$.

$1.4993^{\circ}$ for SBA-16 and in $2 \theta$ of $0.8761^{\circ}, 1.0838^{\circ}$ and $1.4871^{\circ}$ for SBA-16 M. These are attributed to reflection planes (110), (200), (211), respectively, and correspond to the body-centered cubic structure with a $\operatorname{lm} 3 \mathrm{~m}$ spatial group, as reported by Zhao, Huo et al. (1998). Following the modification of mesoporous silica, a decrease in the peak intensities was observed. However, this did not affect the basic structures of SBA-15 M and SBA-16 M. From the diffraction peaks, the interplanar distance of each material was calculated using Bragg's Law $(\mathrm{n} \lambda=2 \mathrm{~d} \sin (\theta))$. In addition, the lattice parameters were also obtained for the materials, and the results were shown in Table 2. After modification, there was a slight increase in the interplanar distance that led to an increase in the parameters, which relates the average diameter of pores with the average thickness of pore walls.

Fig. S2(A) and S2(C) shows the $\mathrm{N}_{2}$ phisiosorption isotherms at $77 \mathrm{~K}$ for SBA. The samples are type IV isotherms: type $\mathrm{H} 1$ hysteresis for SBA15 and SBA-15 M and type H2 hysteresis for SBA-16 and SBA-16 M. According to IUPAC classification all of them are characteristic isotherms of mesoporous materials (Thommes et al., 2015). Fig. S2(B) and S2(D) show the pore size distribution curves obtained by the BJH method. SBA-16 M and SBA-16 exhibited the same pore distribution profile. However, SBA-15 M and SBA-15 showed slightly lower pore sizes, indicating that the presence of organic groups on the SBA-15 surface decreased pore size distribution.

The amount of $\mathrm{N}_{2}$ adsorbed on activated samples decreased, suggesting textural changes. This argument can be strengthened considering the values of specific area, total pore volume, micro and mesoporous volumes, and average pore diameter (Table 2). There was a decrease in the specific area $\left(\mathrm{S}_{\mathrm{BET}}\right)$ of $23.7 \%$ for SBA-15 $\mathrm{M}$ and of $28.75 \%$ for SBA-16 M. The total pore volumes were $0.645 \mathrm{~cm}^{3} \mathrm{~g}^{-1}$ for
SBA-15 M, $0.331 \mathrm{~cm}^{3} \mathrm{~g}^{-1}$ for SBA-16 M, $0.783 \mathrm{~cm}^{3} \mathrm{~g}^{-1}$ for SBA-15, and $0.484 \mathrm{~cm}^{3} \mathrm{~g}^{-1}$ for SBA-16. The average pore diameter of SBA-15 samples followed the same tendency upon modification. No changes in pore diameter of SBA-16 and SBA-16 M were observed. The introduction of vinyl group $\left(\mathrm{CH}_{2}=\mathrm{CH}_{2}\right)$ onto mesoporous silica structure changes both the pores and the surface of the materials, leading to a decrease in specific area, total pore volume, and volume of micro and mesoporos (Owens, Han, Sun, \& Mao, 2015). The diameter and mesoporous lattice parameters were used to calculate the average thickness of pore walls (W). Activated samples showed higher $\mathrm{W}$ values, which are a strong evidence of the organic groups on pore walls.

Fig. S3 shows the FTIR spectra of SBA. The band in the $3440-3500 \mathrm{~cm}^{-1}$ was attributed to $\mathrm{Si}-\mathrm{O}$ bond vibrations of silanol groups. In the region from 400 to $1600 \mathrm{~cm}^{-1}$, three bands are observed, $\sim 460 \mathrm{~cm}^{-1}, \sim 810 \mathrm{~cm}^{-1}$ and $\sim 1080 \mathrm{~cm}^{-1}$, which corresponds, respectively, to stretching, bending, and asymmetric vibrations of oxygen atoms in inter-tetrahedron $\mathrm{O}-\mathrm{Si}-\mathrm{O}$. The band at $960 \mathrm{~cm}^{-1}$ is attributed to $\mathrm{Si}-\mathrm{OH}$ stretching frequency, and the band at $1635 \mathrm{~cm}^{-1}$ corresponds to $\mathrm{O}-\mathrm{H}$ bending frequency from adsorbed water. The absorption bands in the region of $3000-1416 \mathrm{~cm}^{-1}$, owing to $-\mathrm{C}-\mathrm{H}$ vibrational modes of vinyl groups, refer to SBA-15 M, and SBA-16 M. The characteristic peak of vinyl group $\left(1605 \mathrm{~cm}^{-1}\right)$ was not evidenced owing to overlapping of the vibrations of absorbed water (Dai, Wang, Chen, Zheng, \& Lu, 2007; Gao et al., 2012; van Girieken, Iglesias, Morales, \& García, 2010).

\subsection{Characterization of ALGMA}

Fig. 2(A) shows the ${ }^{1} \mathrm{H}$ RMN spectra of sodium alginate and ALGMA. The signs observed at $\delta 6.05 \mathrm{ppm}$ and $\delta 5.62 \mathrm{ppm}$ in the ALGMA spectrum are related to vinyl carbon-bonded hydrogen. The signal at $1.95 \mathrm{ppm}$ was attributed to hydrogen of methyl groups (Guilherme et al., 2010; Wang, Zong, \& Wang, 2013). Fig. 2(B) shows the FTIR spectra of sodium alginate and ALGMA. Both of them showed similar bands at $3500-3400 \mathrm{~cm}^{-1}$ and $2960 \mathrm{~cm}^{-1}$, which are characteristic frequencies of hydroxyl groups $(-\mathrm{OH})$ and $-\mathrm{C}-\mathrm{H}$ bonds of methyl groups, respectively. The bands at $1612 \mathrm{~cm}^{-1}$ and $1420 \mathrm{~cm}^{-1}$ are both attributed to symmetric and asymmetric stretching of carboxylic groups $\left(-\mathrm{COO}^{-}\right)$. The band at $1030 \mathrm{~cm}^{-1}$ is typical of glycoside bonds of sodium alginate $(\mathrm{C}-\mathrm{O}-\mathrm{C})$ and the band at $820 \mathrm{~cm}^{-1}$ refers to bending frequency of $\mathrm{C}-\mathrm{H}$ bonds in mannuronic acid residues (Guilherme et al., 2005; Wang et al., 2013). For ALGMA, two absorption bands were observed at $1714 \mathrm{~cm}^{-1}$ and $1176 \mathrm{~cm}^{-1}$, which were attributed to stretching frequencies of $\mathrm{C}=\mathrm{O}$ and $\mathrm{C}-\mathrm{O}$ bonds of conjugated esters, respectively (Guilherme et al., 2005; Martínez-Gómez, Guerrero, Matsuhiro, \& Pavez, 2017). 

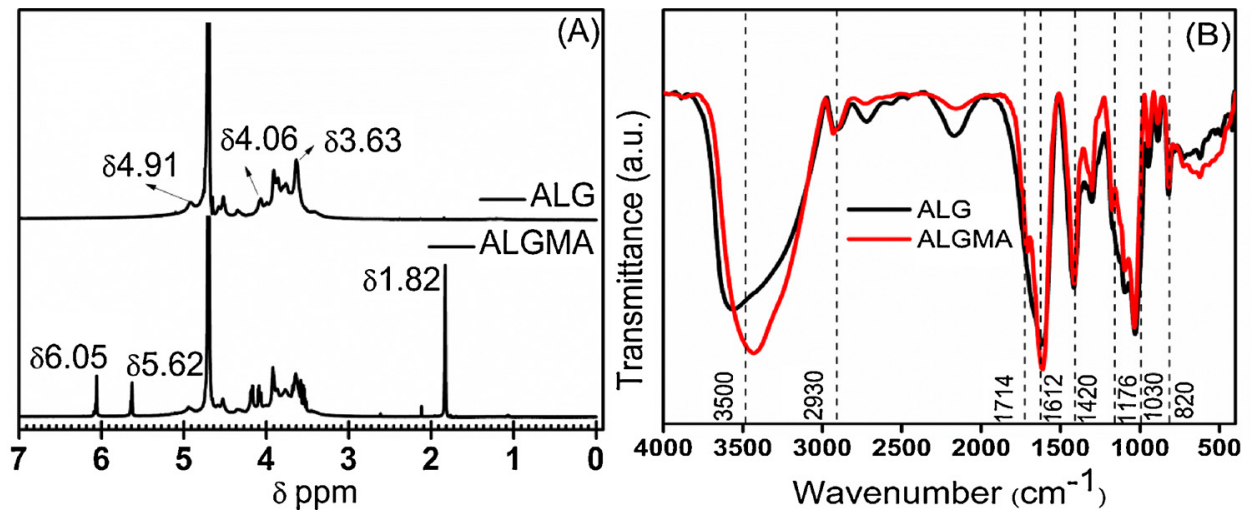

Fig. 2. (A) ${ }^{1} \mathrm{H}$ RMN spectra of sodium alginate and ALGMA and (B) FTIR spectra of sodium alginate and ALGMA.

The modification mechanism of polysaccharides with GMA was systematically analyzed in studies by Guilherme et al. (2005), Guilherme et al. (2012) and Silva et al. (2016). The ${ }^{1} \mathrm{H}$ RMN and FTIR spectra showed that it reacts with GMA by epoxide ring-opening mechanism in a forward and irreversible reaction pathway and these results meet with those of the literature.

\subsection{Morphology of HG and nanocomposites}

Fig. 3(A-C) shows SEM micrographs of hydrogels and nanocomposites fractured in $\mathrm{N}_{2}$ liquid, swollen until equilibrium under $\mathrm{pH}$ 7.4. All samples showed highly porous structure. The fractures revealed an internal structure with pores interconnected; this has particular relevance to the diffusion of water and drugs within the polymer network and such information is of great utility to engineer polymer devices for drug release (Lima-Tenório et al., 2015). To verify the stability of polymer/ silica structure, the presence of mesoporous silica was investigated by swelling HG for long time. EDS analysis of Fig. 3(D) showed Si relative signal at $1.74 \mathrm{keV}$. It makes clear the fact that the SBA was not lost over swelling, indicating possible interactions between silica and polymer.

\subsection{Mechanical behavior of HGs}

Long-term applications require the use of mechanically resistant devices to ensure effective therapy (Gaharwar, Rivera, Wu, \& Schmidt, 2011). Seeking to meet the mechanical requirements for intended application, the samples were analyzed in terms of elasticity moduli (E), which give relevant insight on material resistance. The elasticity moduli indicate that the material experience a reversible deformation when applied stress is removed. The deformation is accommodated by the polymeric chains that tend to reorganize themselves to their initial position (Gaharwar, Rivera et al., 2011), owing to attractive forces. The
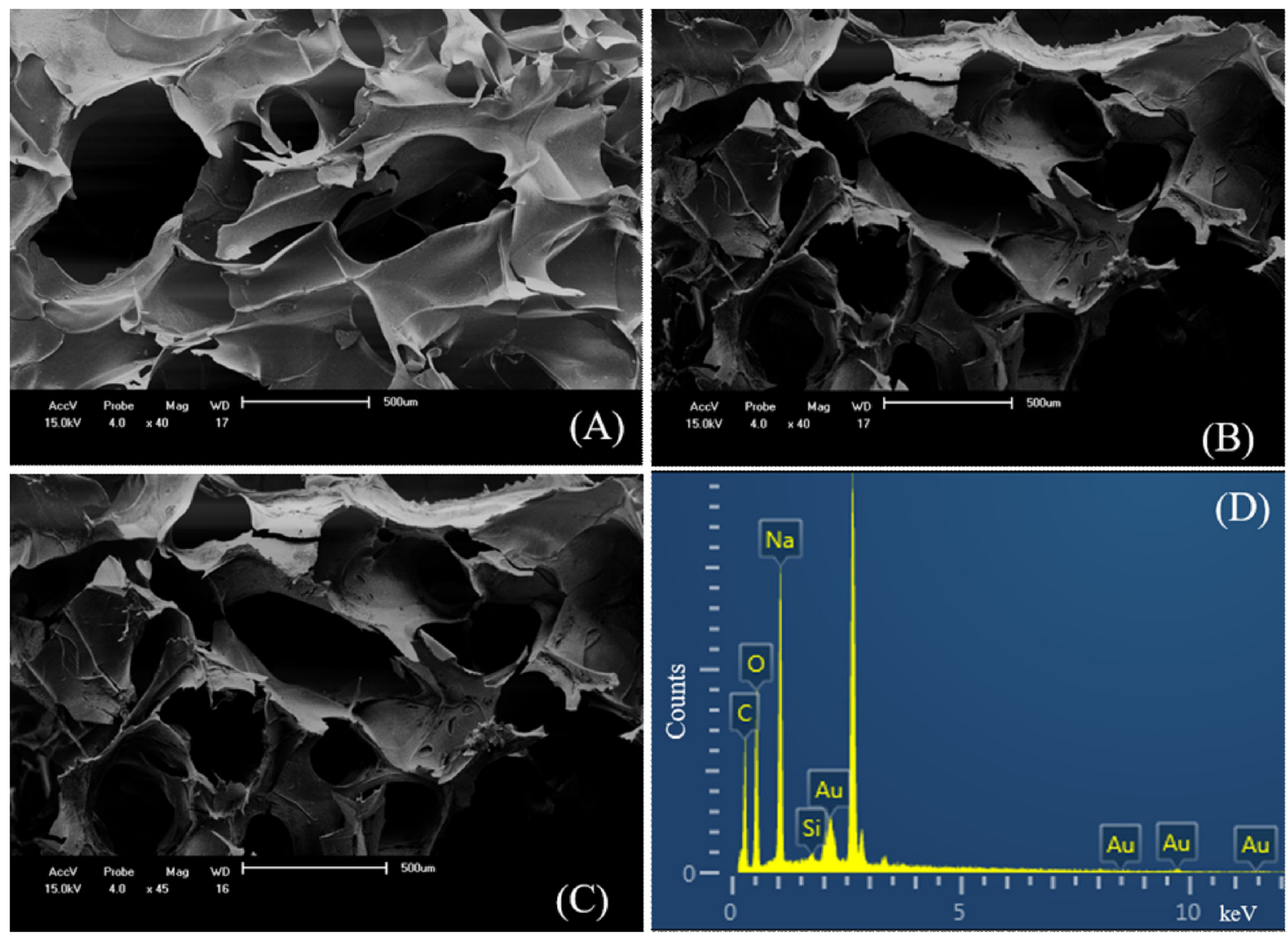

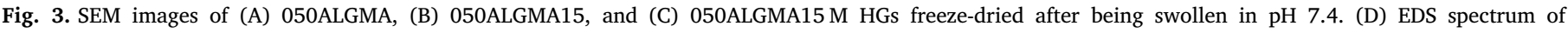
050ALGMA15 M. 
Table 3

Moduli of elasticity (E) of HGS and nanocomposites.

\begin{tabular}{lc}
\hline Hydrogels/Nanocomposites & $\mathrm{E}\left(\mathrm{x} 10^{-3} \mathrm{MPa}\right)$ \\
\hline 025ALGMA & $55.0 \pm 0.94$ \\
025ALGMA15 & $52.0 \pm 0.33$ \\
025ALGMA15M & $67.7 \pm 0.68$ \\
025ALGMA16 & $117.6 \pm 1.2$ \\
025ALGMA16M & $69.0 \pm 0.52$ \\
050ALGMA & $183.4 \pm 3.6$ \\
050ALGMA15 & $356.1 \pm 7.9$ \\
050ALGMA15M & $118.4 \pm 2.9$ \\
050ALGMA16 & $237.9 \pm 5.3$ \\
050ALGMA16M & $210.0 \pm 5.7$ \\
\hline
\end{tabular}

values of $\mathrm{E}$ were obtained through the correlation of stress and strain using Eq. (2) and the fitting parameters were shown in Table 3. HGs without silica showed an increase in $\mathrm{E}$ as the amount of ALGMA increased, which is result of a more cross-linked, less soft structure. The nanocomposites proved to be more rigid because a loader was necessary to compress them to $1 \mathrm{~mm}$.

\subsection{Swelling kinetics}

The samples were swollen in buffer solutions with $\mathrm{pH}$ of 3.5, 5.0 and 7.4 at $37^{\circ} \mathrm{C}$ and the experimental data were correlated to the equilibrium swelling degree (\%) (Fig. S4A and S4B). This approach allows us to evaluate capacity of $\mathrm{HG}$ and nanocomposites to absorb and retain water in dependence of $\mathrm{pH}$ and amounts of ALGMA and mesoporous silica. SW decreased with the increase of ALGMA amount, which is related to a more densely cross-linked network that makes HG expansion more difficult.

The nanocomposites showed lower swelling potential. The networktethered SBA played an important role in restricting the movement of polymeric chains, changing diffusion characteristics of water into HGs. The reduced water absorption capacity can be related to interactions between SBA and polymers, which was also observed in HGs with included clay-minerals (Azmi et al., 2016). It was also observed that SBA-15 M and SBA-16 M can act as a hydrophobic cross-linking agent, thus reducing water absorption (Torres, Urbano, Campos, \& Rivas, 2015).

Swelling tests in different $\mathrm{pH}$ provide valuable information towards understanding the transport water properties in response to changes in the surrounding environment. The HGs and nanocomposites showed reversible swelling/shrinking behavior in response to changes in $\mathrm{pH}$ because of the ionization of carboxylic groups. Upon exposure to alkaline environment, such groups are negatively charged $\left(\mathrm{OH}^{-}\right.$in the surrounding liquid withdraws $\mathrm{H}^{+}$from $\mathrm{COOH}$ ) generating anion-anion electrostatic repulsion forces. As a result, the distance between the polymer chains increases so that water can diffuse into matrix more easily. In the acidic environment, the negative charges on carboxylic group are neutralized (to some extension) with $\mathrm{H}^{+}$decreasing the repulsion forces (Rashidzadeh, Olad, Salari, \& Reyhanitabar, 2014).

\subsection{Studies on water transport}

To have additional insight into swelling mechanism, it is important to understand how water is transported through HG. Semi-empiric Korsmeyer-Peppas model (Eq. (3)) describes the first $60 \%$ of absorbed water, when diffusion of the liquid into HG linearly varies with time

$\frac{M_{t}}{M_{\infty}}=k t^{n}$

where $n$ represents the diffusion coefficient that indicates the specific mechanism of water transport, $\mathrm{k}$ is a parameter related to the diffusion coefficient. $M_{t}$ e $M_{\infty}$ represent the water masses absorbed by HG in a certain time $t$ and in equilibrium, respectively. (Siepmann \& Peppas,
2001).

An alternative useful tool that describes the overall water absorption profile is Weibull empirical function (Eq. (4)).

$\frac{M_{t}}{M_{\infty}}=1-e^{-\left[k_{w}\left(t-\tau_{0}\right)\right] d}$

in which $\tau_{0}$ represents latent time before swelling, $\mathrm{d}$ is related to the mechanism, and $\mathrm{k}_{\mathrm{w}}$ is connected to the constant swelling rate. Eq. (4) represents the diffusion mechanism and swelling rate (Papadopoulou, Kosmidis, Vlachou, \& Macheras, 2006, Tenório-Neto et al., 2015). Adjusting parameters are described in Table S1.

Parameter $n$ has the following conceptual meanings: when $n=0.5$, the swelling mechanism is considered as a Fickian transport. This mechanism suggests that the diffusion rate of the solvent is slower than the relaxation rate. If $\mathrm{n}=1$, the mechanism is defined as case II transport, which means that the diffusion is faster than the relaxation. When $0.5<\mathrm{n}<1$ indicates the existence of an intermediary state between diffusion and relaxation. The HGs and nanocomposites showed values of $\mathrm{n}<0.5$, which suggests pseudo-Fickian water diffusion mechanism. Such a mechanism is found when the time needed to achieve equilibrium swelling is very long (Curcio et al., 2013; Tenório-Neto et al., 2015). Parameter $\mathrm{k}_{\mathrm{w}}$ from Weibull model is associated with the time in which HG reaches the equilibrium swelling. In Table S1 it is observed that the values of $k_{\mathrm{w}}$ were higher in the acidic environment than in the alkaline medium, indicating that the equilibrium is reached earlier. As a general rule, the absorption water mechanism of the hydrogels was shown to be pseudo-Fickian, independently of particle size and pore size of different mesoporous silica.

\subsection{Prednisolone release}

Fig. 4 shows the prednisolone release from HGs and nanocomposites in $\mathrm{pH}$ 7.4. HGs showed higher burst release at the first $10 \mathrm{~min}$, as shown in Table 4. These systems keep the same drawbacks as most polymer hydrogels. This means a high level of prematurely released drug. The addition of SBA to HG was shown to be a determining factor in increasing release time. Note that the initial burst was strongly inhibited and the prednisolone was released with minimal burst kinetics. The 025ALGMA and 050ALGMA HGs released $90 \%$ of prednisolone $\left(\mathrm{t}_{90 \%}\right)$ in 4680 and $3000 \mathrm{~min}$, respectively. The nanocomposites released $90 \%$ of prednisolone within $8000 \mathrm{~min}$. In these systems a longer time was needed for drug release being exhausted. Data of $\mathrm{k}_{\mathrm{w}}$ (Eq. (4)) supported such findings providing additional insight into burst release. Upon addition of SBA there was a decrease in values of $\mathrm{k}_{\mathrm{w}}$, which was associated with the reduction of initial burst release, given that $\mathrm{k}_{\mathrm{w}}$ is related to the constant of release velocity (Silva et al., 2016).

Further analysis of kinetic release was carried out considering the parameter $\mathrm{n}$ as a useful tool to clarify mechanisms that regulate the release extent (Table 4). The prednisolone release mechanism from 025ALGMA and 050ALGMA (without SBA) is governed by case II transport (coupling of Fickian diffusion and macromolecular relaxation) and anomalous transport (macromolecular relaxation), respectively. The nanocomposites showed an anomalous mechanism but with a tendency to a pseudo-Fickian behavior. The reasons for this: (i) SBA weakened the movements of polymer chains, affecting macromolecular relaxation and (ii) the distribution of the nanoparticles within HG makes the release into surrounding liquid more resistant (tortuosity effect), playing a role as a retardant factor for release.

The prednisolone loading efficiency of hydrogels was shown to be between 48 a $97 \%$ (Table 4). The hydrogels showed higher drug loading efficiency influenced by the increase in the amount of alginate and silica. However, the prednisolone release rate from these hydrogels was shown to be slower, providing a steady release into the solution. 

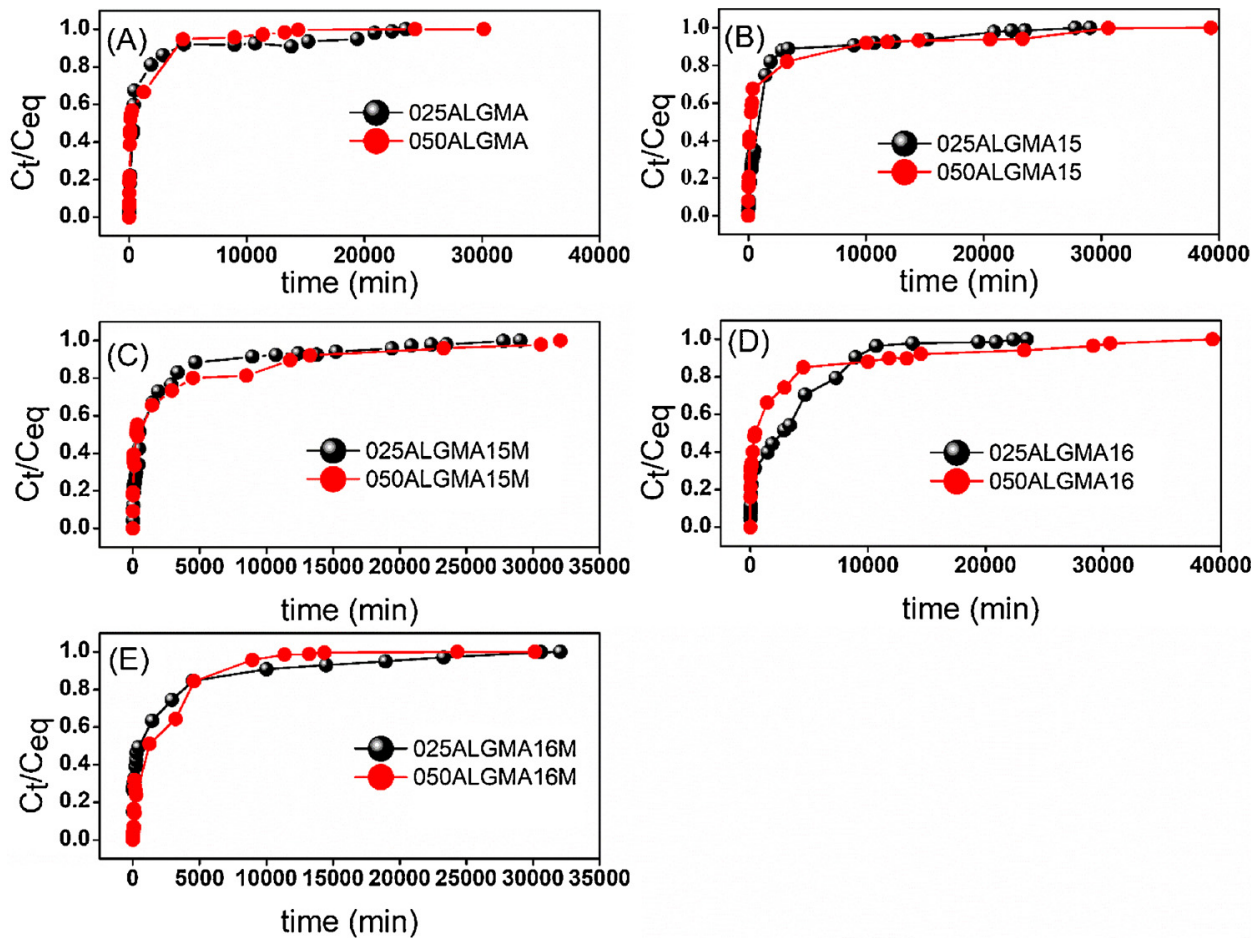

Fig. 4. Prednisolone release from (A) HGs and nanocomposites (B) SBA-15, (C) SBA-15 M, (D) SBA-16 and (E) SBA-16 M.

\subsection{Cytotoxicity evaluation}

Cell viability analysis is a useful tool to analyze spontaneous cell death after being exposed to different experimental conditions. In this sense, dye exclusion tests are used to determine the number of live and dead cells for evaluating cytotoxicity in experimental investigations. One of them is the Trypan Blue (TB) assay, which is based on the principle that intact plasma membranes in live cells exclude this dye, whereas dead cells do not (Fig. 5A). The cell line used in this study was a human non-malignant immortalized RWPE-1 prostatic epithelial cell line that was derived from the peripheral zone of a histologically normal adult human prostate. This epithelial cell line is adherent and easily cultivated in K-SFM following the manufacturer's instructions. Cell culture allowed us to evaluate the toxicological risk of the synthetized materials using in vitro application. In combination, those two tools showed the biocompatibility of HG, SBA alone or in association. After $48 \mathrm{~h}$ of incubation in the presence of two different concentrations of the materials no changes in rate of cell viability of human prostatic epithelial cells were observed (Fig. 5B and C).

\section{Conclusions}

HG nanocomposites based on alginate and mesoporous silica with reduced release burst and enhanced elastic moduli were successfully developed. These effects are results of long-lasting entrapment of SBA15 and SBA-16 within HG that regulated release mechanism and improved gel properties. In these systems, the release of prednisolone was slower and the burst release was reduced by $90 \%$, given that the drug diffuses through a longer path from nanocomposite into surrounding solution. They showed an accentuated increase in elastic moduli, proving to be more rigid than pure HGs. Cell culture and cytotoxicity assay results were quite consistent. The large number of cells evaluated provides reliable results regarding the real amount of dead and live cells exposed to the different synthetized nanocomposites, which can be a useful tool for further physiological and pathological applications, like drug-delivery device.

\section{Acknowledgments}

The authors thank both the COMCAP - UEM for SEM analyses and the Brazilian agencies for fellowship CNPQ (Process: 577527/2008-8, 310820/2011-1, 101157/2016-9), Fundação Araucária/PR, Brazil

Table 4

Fitting parameters of Eqs. (3) and (4) to release kinetics of HGs and nanocomposites prepared in different synthesis compositions.

\begin{tabular}{|c|c|c|c|c|c|}
\hline $\begin{array}{l}\text { HGs/ } \\
\text { nanocomposites }\end{array}$ & $\mathrm{n}$ & $\mathrm{k}_{\mathrm{w}}\left(\min ^{-1}\right)$ & $\mathrm{t}_{10 \min }(\%)$ & $\mathrm{t}_{90 \%}(\mathrm{~min})$ & Loading efficiency (\%) \\
\hline 025ALGMA & $0.89 \pm 0.03$ & $0.002 \pm 5.1 \mathrm{E}^{-4}$ & 2.5 & 4680 & 54.85 \\
\hline 025ALGMA15 & $0.66 \pm 0.01$ & $9.64 \mathrm{E}^{-4} \pm 4.9 \mathrm{E}^{-5}$ & 2.5 & 8960 & 49.82 \\
\hline 025ALGMA15M & $0.59 \pm 0.06$ & $8.75 \mathrm{E}^{-4} \pm 6.9 \mathrm{E}^{-5}$ & 3.9 & 8960 & 48.40 \\
\hline 025ALGMA16 & $0.58 \pm 0.07$ & $1.41 \mathrm{E}^{-4} \pm 6.3 \mathrm{E}^{-5}$ & 4.3 & 8960 & 74.28 \\
\hline 025ALGMA16M & $0.21 \pm 0.01$ & $5.4 \mathrm{E}^{-4} \pm 1.4 \mathrm{E}^{-5}$ & 15.1 & 10020 & 70.35 \\
\hline 050ALGMA & $0.82 \pm 0.01$ & $0.0025 \pm 6.2 \mathrm{E}^{-4}$ & 5.54 & 3000 & 67.80 \\
\hline 050ALGMA15 & $0.57 \pm 0.02$ & $0.003 \pm 3.0 \mathrm{E}^{-4}$ & 7.9 & 10020 & 76.16 \\
\hline 050ALGMA15M & $0.44 \pm 0.07$ & $9.8 \mathrm{E}^{-4} \pm 4.4 \mathrm{E}^{-5}$ & 9.0 & 11800 & 88.11 \\
\hline 050ALGMA16 & $0.25 \pm 0.01$ & $7.4 \mathrm{E}^{-4} \pm 1.3 \mathrm{E}^{-5}$ & 16.15 & 14490 & 96.54 \\
\hline 050ALGMA16M & $0.77 \pm 0.09$ & $4.8 \mathrm{E}^{-4} \pm 1.0 \mathrm{E}^{-5}$ & 2.1 & 6000 & 70.90 \\
\hline
\end{tabular}



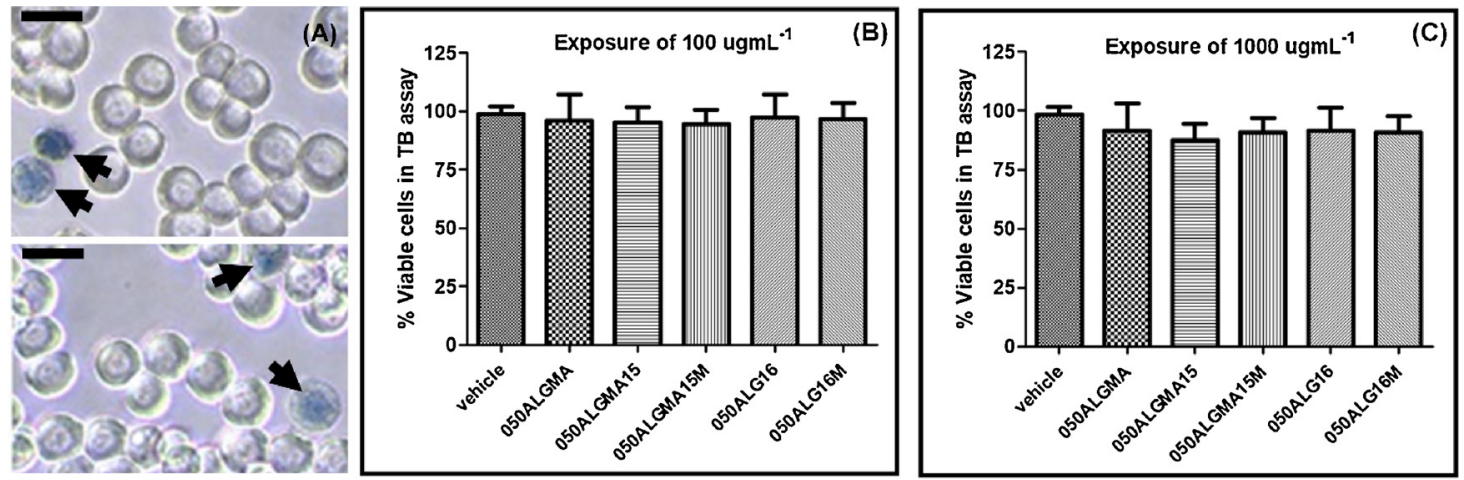

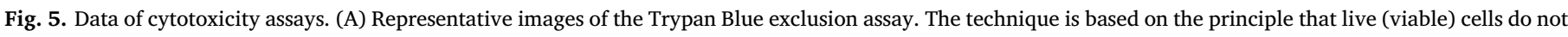

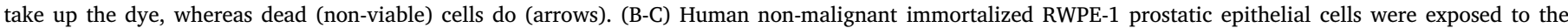

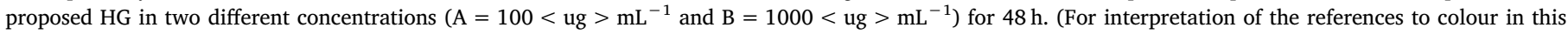
figure legend, the reader is referred to the web version of this article.)

(Process: 830/2013), and CAPES by financial support.

\section{Appendix A. Supplementary data}

Supplementary data associated with this article can be found, in the online version, at https://doi.org/10.1016/j.carbpol.2018.04.107.

\section{References}

Ahmed, E. M. (2015). Preparation, characterization, and applications. Journal of Advanced Research, 6, 105-121.

Azmi, S., Razak, S. I. A., Kadir, M. R. A., Iqbal, N., Hassan, R., Nayan, N. D. M., et al. (2016). Reinforcement of poly(vinyl alcohol) hydrogel with halloysite nanotubes as potential biomedical materials. Soft Mater, 15, 45-54.

Bastakoti, B. P., Hsu, Y., Liao, C., Wu, S. H., Inoue, K. C. W., Yusa, M., et al. (2013). Inorganic-organic hybrid nanoparticles with biocompatible calcium phosphate thin shells for fluorescence enhancement. Chemistry an Asian Journal, 8, 1301-1305.

Boza, A. F., Kupfer, V. L., Oliveira, A. R., Radovanovic, E., Rinaldi, A. W., Meneguin, J. G., et al. (2016). Synthesis of a-aminophosphonates using a mesoporous silica catalyst produced from sugarcane bagasse ash. RSC Advances, 6, 23981-23986.

Chen, H. W., Chiang, Y. D., Kuang, C. W., Sakai, N., Ikegami, M., Yamauchi, Y., et al. (2014). Highly efficient plastic-based quasi-solid-state dye-sensitized solar cells with light-harvesting mesoporous silica nanoparticles gel-electrolyte. Journal of Power Sources, 245, 411-417.

Curcio, M., Altimari, I., Spizzirri, U. G., Cirillo, G., Vittorio, O., Puoci, F., et al. (2013). Biodegradable gelatin-based nanospheres as $\mathrm{pH}$-responsive drug delivery systems. Journal of Nanoparticle Research, 15, 1581-1592.

Dai, Q., Wang, X., Chen, G., Zheng, Y., \& Lu, G. (2007). Direct synthesis of Cerium(III) incorporated SBA-15 mesoporous molecular sieves by two-step synthesis method. Microporous Mesoporous Materials, 100, 268-275.

Din, F., Mustapha, O., Kim, D. W., Rashid, R., Park, J. H., Choi, J. Y., et al. (2015). Novel dual-reverse thermosensitive solid lipid nanoparticle-loaded hydrogel for rectal administration of flurbiprofen with improved bioavailability and reduced initial burst effect. European Journal of Pharmaceutics and Biopharmaceutics, 94, 64-72.

Elumalai, V., \& Dharmalingam, S. (2016). Synthesis characterization and performance evaluation of ionic liquid immobilized SBA-15/quaternised polysulfone composite membrane for alkaline fuel cell. Microporous Mesoporous Materials, 236, 260-268.

Gaharwar, A. K., Rivera, C., Wu, C. J., Chan, B. K., \& Schmidt, G. (2013). Photocrosslinked nanocomposite hydrogels from PEG and silica nanospheres: Structural, mechanical and cell adhesion characteristics. Materials Science and Engineering: C, 33, 1800-1807.

Gaharwar, A. K., Dammu, S. A., Canter, J. M., Wu, C. J., \& Schmidt, G. (2011). Highly extensible, tough, and elastomeric nanocomposite hydrogels from poly(ethylene glycol) and hydroxyapatite nanoparticles. Biomacromolecules, 12, 1641-1650.

Gaharwar, A. K., Rivera, C. P., Wu, C. J., \& Schmidt, G. (2011). Transparent, elastomeric and tough hydrogels from poly(ethylene glycol) and silicate nanoparticles. Acta Biomaterialia, 7, 4139-4148.

Gao, Z., Zhan, W., Wang, Y, Guo, Y., Wang, L., Guo, Y, et al. (2012). Aldehyde-functionalized mesostructured cellular foams prepared by copolymerization method for immobilization of penicillin G acylase. Microporous Mesoporous Materials. 202, 90-96.

García-Astrain, C., Chen, C., Burón, M., Palomares, T., Eceiza, A., Fruk, K., et al. (2015). Biocompatible hydrogel nanocomposite with covalently embedded silver nanoparticles. Biomacromolecules, 16, 1301-1310.

Ghorpade, V. S., Yadav, A. V., \& Dias, R. J. (2016). Citric acid crosslinked cyclodextrin/ hydroxypropylmethylcellulose hydrogel films for hydrophobic drug delivery. International Journal of Biological Macromolecules, 93, 75-86.

Guilherme, M. R., Reis, A. V., Takahashi, S. H., Rubira, A. F., Feitosa, J. P. A., \& Muniz, E. C. (2005). Synthesis of a novel superabsorbent hydrogel by copolymerization of acrylamide and cashew gum modified with glycidyl methacrylate. Carbohydrate
Polymers, 61, 464-471.

Guilherme, M. R., Fajardo, A. R., Moia, T. A., Kunita, M. H., Gonçalves, M. C., Rubira, A. F., et al. (2010). Porous nanocomposite hydrogel of vinyled montmorillonite-crosslinked maltodextrin-co-dimethylacrylamide as a highly stable polymer carrier for controlled release systems. European Polymer Journal, 46, 1465-1474.

Guilherme, M. R., Oliveira, R. S., Mauricio, M. R., Cellet, T. S. P., Pereira, G. M., Kunita, M. H., et al. (2012). Albumin release from a brain-resembling superabsorbent magnetic hydrogel based on starch. Soft Matter, 8, 6629-6637.

Hashemikia, S., Hemmatinejad, N., Ahmadi, E., \& Montazer, M. (2015). Optimization of tetracycline hydrochloride adsorption on amino modified SBA-15 using response surface methodology. Journal of Colloid and Interface Science, 43, 105-114.

Hezaveh, H., \& Muhamad, I. I. (2013). Effect of MgO nanofillers on burst release reduction from hydrogel nanocomposites. Journal of Materials Science: Materials in Medicine, 24, 1443-1453.

Hoare, T. R., \& Kohane, D. S. (2007). Hydrogels in drug delivery: Progress and challenges. Polymer, 49, 1993-2007.

Hou, R., Nie, L., Du, G., Xiong, X., \& Fu, J. (2015). Natural polysaccharides promote chondrocyte adhesion and proliferation on magnetic nanoparticle/PVA composite hydrogels. Colloids and Surfaces B: Biointerfaces, 132, 146-154.

Huang, W. C., Liu, K. H., Liu, T. C., Liu, D. M., \& Chen, S. Y. (2014). Synergistic hierarchical silicone-modified polysaccharide hybrid as a soft scaffold to control cell adhesion and proliferation. Acta Biomaterialia, 10, 3546-3556.

Huang, Y., Yao, M., Zheng, X., Liang, X., Su, X., Zhang, Y., et al. (2015). Effects of chitin whiskers on physical properties and osteoblast culture of alginate based nanocomposite hydrogels. Biomacromolecules, 16, 3499-3507.

Iviglia, G., Cassinelli, C., Torres, E., Baino, F., Morra, M., \& Vitale-Brovarone, C. (2016). Novel bioceramic-reinforced hydrogel for alveolar bone regeneration. Acta Biomaterialia, 44, 97-109.

Kamachi, Y., Bastakoti, B. P., Alshehri, S. M., Miyamoto, N., Nakato, T., \& Yamauchi, Y. (2016). Thermo-responsive hydrogels containing mesoporous silica toward controlled and sustainable releases. Materials Letter, 168, 176-179.

Kerh, N. S. (2016). Enantiomorphous periodic mesoporous organosilica-based nanocomposite hydrogel scaffolds for cell adhesion and cell enrichment. Biomacromolecules, 17, 1117-1122.

Lian, H.-Y., Liang, Y.-H., Yamauchi, Y., \& Wu, K. C.-W. (2011). A hierarchical study on load/release kinetics of guest molecules into/from mesoporous silica thin films. The Journal of Physical Chemistry C, 2011, 6581-6590.

Liang, Y. H., Liu, C. H., Liao, S. H., Lin, Y. Y., Tang, H. W., Liu, S. Y., et al. (2012). Cosynthesis of cargo-loaded hydroxyapatite/alginate core-shell nanoparticles (HAP@Alg) as pH-responsive nanovehicles by a pregel method. Applied Materials \& Interfaces, 4, 6720-6727.

Liao, S. H., Liu, C. H., Bastakoti, B. P., Suzuki, N., Chang, Y., Yamauchi, Y., et al. (2015), Functionalized magnetic iron oxide/alginate coreshell nanoparticles for targeting hyperthermia. Internacional Journal of Nanomedicine, 10, 3315-3328.

Lima-Tenório, M. K., Tenório-Neto, E. T., Guilherme, M. R., Garcia, F. P., Nakamura, C. V., Pineda, E. A. G., et al. (2015). Water transport properties through starch-based hydrogel nanocomposites responding to both $\mathrm{pH}$ and a remote magnetic field. Chemical Engineering Journal, 259, 620-629.

Manzano, M., Aina, V., Areán, C. O., Balas, F., Cauda, V., Colilla, M., et al. (2008). Studies on MCM-41 mesoporous silica for drug delivery: Effect of particle morphology and amine functionalization. Chemical Engineering Journal, 137, 30-37.

Martínez-Gómez, F., Guerrero, J., Matsuhiro, B., \& Pavez, J. (2017). In vitro release of metformin hydrochloride from sodium alginate/polyvinyl alcohol hydrogels. Carbohydrate Polymers, 155, 182-191.

Muniz, E. C., \& Geuskens, G. (2001). Compressive elastic modulus of polyacrylamide hydrogels and semi-IPNs with poly(N-isopropylacrylamide). Macromolecules, 34, 4480-4484.

Owens, D., Han, A., Sun, L., \& Mao, Y. (2015). Synthesis of VTMS(X)-HMS-3 mesoporous ordered silica for hydrogen storage. International Journal of Hydrogen Energy, 40, 2736-2741.

Panic, V. V., Spasojevic, P. M., Radoman, T. S., Dzunuzovic, E. S., Popovic, I. G., \& 
Velickoiv, S. J. (2015). Methacrylic acid based polymer networks with a high content of unfunctionalized nanosilica: Particle distribution, swelling. and Rheological Properties The Journal of Physical Chemistry C, 119, 610-622.

Papadopoulou, V., Kosmidis, K., Vlachou, M., \& Macheras, P. (2006). On the use of the Weibull function for the discernment of drug release mechanisms. International Journal of Pharmaceutics, 309, 44-50.

Poels, J., Abou-Ghannam, G., Decamps, A., Leyman, M., Rieux, A., \& Wyns, C. (2016). Transplantation of testicular tissue in alginate hydrogel loaded with VEGF nanoparticles improves spermatogonial recovery. Journal Controlled Release, 234, 78-89.

Rashidzadeh, A., Olad, A., Salari, D., \& Reyhanitabar, A. (2014). On the preparation and swelling properties of hydrogel nanocomposite based on Sodium alginate-g-Poly (acrylic acid-co-acrylamide)/Clinoptilolite and its application as slow release fertilizer. Journal of Polymer Research, 21, 2-15.

Reis, A. V., Farjado, A. R., Schuquel, I. T. A., Guilherme, M. R., Vidotti, G. J., Rubira, A. F., et al. (2009). Reaction of glycidyl methacrylate at the hydroxyl and carboxylic groups of poly(vinyl alcohol) and poly(acrylic acid): Is this reaction mechanism still unclear? The Journal of Organic Chemistry, 74, 3750-3757.

Sevimli, F., \& Yilmaz, A. (2012). Surface functionalization of SBA-15 particles for amoxicillin delivery. Microporous Mesoporous Materials, 158, 281-291.

Shah, A. T., Din, M. I., Kanwal, F. N., \& Mirza, M. L. (2015). Direct synthesis of mesoporous molecular sieves of Ni-SBA-16 by internal $\mathrm{pH}$ adjustment method and its performance for adsorption of toxic Brilliant Green dye. Arabian Journal of Chemistry, 8, 579-586.

Siepmann, J., \& Peppas, N. A. (2001). Modeling of drug release from delivery systems based on hydroxypropyl methylcellulose (HPMC). Advanced Drug Delivery Reviews, 48, 139-157.

Silva, E. P., Guilherme, M. R., Garcia, F. P., Nakamura, C. V., Cardozo-Filho, L., Alonso, C. G., et al. (2016). Drug release profile and reduction in the in vitro burst release from pectin/HEMA hydrogel nanocomposites crosslinked with titania. RSC Advances, 6, 19060-19068.

Tenório-Neto, E. T., Guilherme, M. R., Lima-Tenório, M. K., Scariot, D. B., Nakamura, C. V., Rubira, A. F., et al. (2015). Synthesis and characterization of a pH-responsive poly (ethylene glycol)-based hydrogel: Acid degradation, equilibrium swelling, and absorption kinetic characteristics. Colloid and Polymer Science, 293, 3611-3622.

Thommes, M., Kanelo, K., Neimark, A. V., Olivier, J. P., Rodriguez-Reinoso, F.,
Rouquerol, J., et al. (2015). Physisorption of gases, with special reference to the evaluation of surface area and pore size distribution (IUPAC Technical Report). International Union of Pure Ad Applied Chemistry, 87, 1051-1069.

Topuz, F., Bartneck, M., Pan, Y., \& Tacke, F. (2017). One-Step fabrication of biocompatible multifaceted nanocomposite gels and nanolayers. Biomacromolecules, 18, 386-397.

Torres, C. C., Urbano, B. F., Campos, C. H., \& Rivas, B. L. (2015). Composite hydrogel based on surface modified mesoporous silica and poly[(2-acryloyloxy)ethyl trimethylammonium chloride. Materials Chemistry and Physics, 152, 69-76.

van Girieken, R., Iglesias, J., Morales, V., \& García, R. A. (2010). Synthesis and characterization of SBA-15 materials functionalized with olefinic groups and subsequent modification through oxidation procedures. Microporous Mesoporous Materials, 131, 321-330.

Wang, Q., Mynar, J. L., Yoshida, M., Lee, E., Lee, M., Okuro, K., et al. (2010). High-watercontent mouldable hydrogels by mixing clay and a dendritic molecular binder. Nature, 463, 339-343.

Wang, W., Zong, L., \& Wang, A. (2013). A nanoporous hydrogel based on vinyl-functionalized alginate for efficient absorption and removal of $\mathrm{Pb}^{2+}$ ions. International Journal of Biological Macromolecules, 62, 225-231.

Yadollahi, M., Farhoudian, S., Barkhordari, S., Gholamali, I., Farhadnejad, H., \& Motasadizadeh, H. (2016). Facile synthesis of chitosan/ZnO bio-nanocomposite hydrogel beads as drug delivery systems. International Journal of Biological Macromolecules, 82, 273-278.

Zare-Akbari, Z., Farhadnejad, H., Furughi-Nia, B. H., Abedin, S., Yadollahi, M., \& Khorsand-Ghayeni, M. (2016). PH-sensitive bionanocomposite hydrogel beads based on carboxymethyl cellulose/ZnO nanoparticle as drug carrier. International Journal of Biological Macromolecules, 93, 1317-1327.

Zhao, D., Feng, J., Huo, Q., Melosh, N., Fredrickson, G. H. B., Chmelka, F., et al. (1998) Triblock Copolymer Syntheses of Mesoporous Silica with Periodic 50-300 Angstrom Pores. Science, 279, 548-552.

Zhao, D., Huo, Q., Feng, J., Chmelka, B. F., \& Stucky, G. D. (1998). Nonionic triblock and star diblock copolymer and oligomeric surfactant syntheses of highly ordered, hydrothermally stable, mesoporous silica structures. Journal of the American Chemical Society, 120, 6024-6036. 